\title{
Experiencia docente en un curso de matemáticas virtual en pandemia covid -19
}

\author{
Judith Rocha Gámez \\ judith.rochagm@uanl.edu.mx \\ Samantha Armijo Martinez \\ sarmijo.me5021@uanl.edu.mx \\ Departamento de Química Analítica \\ Facultad de Medicina UANL
}

\section{RESUMEN}

Nuestro compromiso como docentes es desarrollar en nuestros estudiantes competencias que les permitan responder a las exigencias del mercado laboral y social. ¿Entonces qué técnicas y estrategias de enseñanza virtual se deben implementar para que los estudiantes construyan un aprendizaje significativo?

Los docentes tuvimos que modificar nuestros programas, tareas, y formas de impartir la clase de manera virtual. Ante la contingencia, los docentes nos enfrentamos a un nuevo reto: dar clases de manera virtual o virtual-presencial (mixto). Como profesoras de matemáticas nuestro objetivo fue aprovechar la situación para que los estudiantes desarrollaran habilidades — como leer, escribir y hablar matemáticamente — lo que me permitió identificar áreas de oportunidad para trabajar. En el presente trabajo compartimos la experiencia de un curso virtual de matemáticas bajo la modalidad de competencias a nivel licenciatura en la carrera de Químico Clínico Biólogo que se imparte en la facultad de Medicina de la Universidad Autónoma de Nuevo León.

La plataforma Moodle nos sirvió de comunicación y soporte técnico para las actividades ponderables del curso y la plataforma Microsof Teams para las clases virtuales.

Palabras clave. enseñanza; virtual; matemáticas; desarrollo de habilidades 


\title{
Eaching experience in a virtual mathematics course in the covid -19 pandemic
}

\begin{abstract}
ABSTRAC
Our commitment as teachers is to develop in our students skills that allow them to respond to the demands of the labor and social market. So what virtual teaching techniques and strategies need to be implemented for students to build meaningful learning?

Teachers had to modify our programs, tasks, and ways to teach the class virtually. Faced with contingency, teachers face a new challenge: to teach in a virtual or virtual-face-toface (mixed) way. As math teachers, our goal was to take advantage of the situation for students to develop skills — such as reading, writing, and speaking math - that allowed me to identify areas of opportunity to work. In this work we share the experience of a virtual course of mathematics under the modality of competences at the bachelor's level in the career of Clinical Chemistry Biologist that is taught in the faculty of Medicine of the Universidad Autonoma de Nuevo León.

The Moodle platform served as communication and technical support for the weighted activities of the course and the Microsof Teams platform for virtual classes.
\end{abstract}

Keywords. teaching; virtual; math; skill development

Artículo recibido: 10 Agosto. 2021 Aceptado para publicación: 07. Setiembre. 2021 Correspondencia: judith.rochagm@uanl.edu.mx Conflictos de Interés: Ninguna que declarar 


\section{I- INTRODUCCIÓN}

No es novedad que la situación actual no se había previsto, sin embargo, los seres humanos nos adaptamos al cambio, la pandemia prácticamente nos obligó a modificar muchas de nuestras tareas comunes, y una de ellas sin duda fueron las clases presenciales. $(1)(14)$

Nuestro compromiso como docentes es desarrollar en nuestros estudiantes competencias que les permitan responder a las exigencias del mercado laboral y social. ¿Entonces qué técnicas y estrategias de enseñanza virtual se deben implementar para que los estudiantes construyan un aprendizaje significtivo?(3)

Los docentes tuvimos que modificar nuestros programas, tareas, y formas de impartir la clase de manera virtual.(7)(10) Ante la contingencia, los docentes nos enfrentamos a un nuevo reto: dar clases de manera virtual o virtual-presencial (mixto). Como profesoras de matemáticas nuestro objetivo fue aprovechar la situación para que los estudiantes desarrollaran habilidades — como leer, escribir y hablar matemáticamente - lo que me permitió identificar áreas de oportunidad para trabajar.(2)(13) En el presente trabajo compartimos la experiencia de un curso virtual de matemáticas bajo la modalidad de competencias a nivel licenciatura en la carrera de Químico Clínico Biólogo que se imparte en la facultad de Medicina de la Universidad Autónoma de Nuevo León.

La plataforma Moodle nos sirvió de comunicación y soporte técnico para las actividades ponderables del curso y la plataforma Teams para las clases virtuales, el uso de Microsoft Teams pudimos establecer un contacto con los estudiantes ya que al contar con video llamada podemos verlos en tiempo y forma cuando están en la sesión facilitando la retroalimentación hacia una duda o pregunta.

LA EVALUACIÓN EN AMBIENTES VIRTUALES Existen diferentes formas para caracterizar un ambiente virtual. De manera general, un ambiente virtual es el modelo generado y operado en una computadora, que simula o reproduce un entorno real, en sus aspectos más importantes, de acuerdo a la finalidad de su utilización En el campo de la educación, los ambientes virtuales facilitan la comunicación de información textual, auditiva y visual, así como su registro temporal o permanente, de manera sincrónica, o en perfecta correspondencia temporal. Y asincrónica, o sin correspondencia temporal, para a apoyar el aprendizaje a distancia o en línea. Entre las ventajas de este enfoque se identifica a los siguientes autores quienes describen las principales ventajas y desventajas 
de la evaluación en ambientes virtuales:: entornos virtuales de aprendizaje y Incentiva el desarrollo de destrezas importantes en los actuales entornos económicos y sociales, como son la comunicación, el trabajo en equipo y el pensamiento crítico. y Reduce tiempos y costos, al facilitar el uso de técnicas para evaluar grupos más numerosos y diversificados. y Posibilita el desarrollo de nuevas formas de evaluación y su integración con otras actividades del aprendizaje, así como una retroalimentación inmediata de sus resultados. y Ofrece mayores oportunidades para practicar los conocimientos y destrezas adquiridas. y El principal problema de la evaluación en un ambiente virtual o a distancia suele ser el de la fiabilidad, dado que "puede inducir al plagio" (4)

Se realizaron comparaciones entre las calificaciones obtenidas con el grupo del periodo agosto-diciembre del 2020 (clases virtuales) vs dos grupos de años anteriores 2019 y 2018 quienes cursaron la unidad de aprendizaje en forma presencial observando una marcada diferencia

\section{II- PLANTEAMIENTO DEL PROBLEMA}

La dificultad de impartición de la cátedra de matemáticas superior a dos grupos de 40 alumnos cada uno de forma virtual. Se plantea abordar mediante la ejecución de ejercicios en clase, y la explicación de estos y su procedimiento, haciendo uso de plataformas digitales para que la clase sea más atractiva, con participación activa de cada uno de los alumnos.

\section{III- METODOLOGÍA}

Se utilizará la plataforma Microsoft Teams como aula virtual para llevar a cabo las sesiones teóricas del curso de Matemáticas superior que se imparte en el primer semestre de la carrera de QCB con duración de 60 minutos.

En la plataforma Teams se realizó la formación de subgrupos, recordado que los grupos de teoría son de 40 alumnos ( en cada equipo de TEAMS, estos subgrupos o canales se establecieron como "salas" 6 en este caso) en donde al final de cada sesión ( 15 min aprox antes de terminar) se les invitaba a abandonar la sesión general y entrar a su sala de trabajo. Así el docente entraba a cada sala a petición de los alumnos quienes tuvieran alguna duda.

Otra estrategia utilizada fue el recurso digital Nearpood, (12)en donde en esta aplicación el docente fue creando diferentes presentaciones que incluyen diapositivas, actividades interactivas (time to climb) donde se repasaban los conceptos o ejercicios vistos en esa 
sesión, así mismo también se hacía el uso de simuladores para diferentes temas como lo son: ecuación de la recta, despeje de ecuaciones, ecuación cuadrática ente otros. El simulador propone diferentes niveles de complejidad en los procesos de aprendizaje, para que cada estudiante identifique en qué estado se encuentran sus habilidades y conocimientos, busque constructivamente un punto óptimo que lo homogenice con el grupo. (6)

\section{Tabla 1. Programa Analítico de UA matemáticas superior evaluación}

\begin{tabular}{|c|c|c|c|}
\hline \multirow{7}{*}{ Etapa 1} & $\begin{array}{l}\text { Evidencia 1. Examen de resolución de problemas con operaciones aritméticas y algoritmos } \\
\text { algebraicos fundamentales }\end{array}$ & $10 \%$ & \multirow{7}{*}{$36 \%$} \\
\hline & $\begin{array}{l}\text { Evidencia 2. Examen de resolución de problemas con algoritmos algebraicos donde se } \\
\text { involucran ecuaciones }\end{array}$ & $10 \%$ & \\
\hline & 1.1. Cuestionario sobre el contexto de la UA. & $2 \%$ & \\
\hline & 1.2. Ensayo sobre la importancia de la UA en el programa educativo de QCB. & $2 \%$ & \\
\hline & 1.3. Mapas conceptuales indicados por el profesor. & $2 \%$ & \\
\hline & $\begin{array}{l}\text { 1.4. Resolución en el aula de problemas donde se usan operaciones aritméticas y algoritmos } \\
\text { algebraicos. }\end{array}$ & $4 \%$ & \\
\hline & $\begin{array}{l}\text { 1.5. Resolución extra aula de problemas generales y/o del campo de la } \\
\text { química donde se usan operaciones aritméticas y algoritmos algebraicos. }\end{array}$ & $6 \%$ & \\
\hline \multirow{4}{*}{ Etapa 2} & $\begin{array}{l}\text { Evidencia 3. Examen de resolución de problemas con funciones trascendentes geometría } \\
\text { analítica y trigonometría. }\end{array}$ & $10 \%$ & \multirow{4}{*}{$22 \%$} \\
\hline & 2.1. Mapas conceptuales indicados por el profesor. & $2 \%$ & \\
\hline & $\begin{array}{l}\text { 2.2. Resolución en el aula de problemas donde se usa geometría analítica, } \\
\text { trigonometría y funciones logarítmicas y exponenciales. }\end{array}$ & $4 \%$ & \\
\hline & $\begin{array}{l}\text { 2.3. Resolución extra aula de problemas generales y/o del campo de la química donde se } \\
\text { usa geometría analítica, trigonometría y funciones logarítmicas y exponenciales. }\end{array}$ & $6 \%$ & \\
\hline \multirow{4}{*}{ Etapa 3} & $\begin{array}{l}\text { Evidencia 4. Examen de resolución de problemas con pre cálculo cálculo diferencial e } \\
\text { integral. }\end{array}$ & $10 \%$ & \multirow{4}{*}{$22 \%$} \\
\hline & 3.1. Mapas conceptuales indicados por el profesor. & $2 \%$ & \\
\hline & $\begin{array}{l}\text { 3.2. Resolución en el aula de problemas donde se usan limites, continuidad, derivadas, } \\
\text { integrales y ecuaciones diferenciales. }\end{array}$ & $4 \%$ & \\
\hline & $\begin{array}{l}\text { 3.3. Resolución extra aula de problemas generales y/o del campo de la química donde se } \\
\text { usan limites, continuidad, derivadas, integrales y ecuaciones diferenciales. }\end{array}$ & $6 \%$ & \\
\hline
\end{tabular}

Para la explicación de los problemas y su resolución se hizo uso de la aplicación de pizarra en la sesión de Teams 
Las evaluaciones(9) como los Quiz semanales y los exámenes parciales se aplicaron utilizando la plataforma Moodle(11) para resolverlos y Microsof -teams simultáneamente ésta última para que mantuvieran sus cámaras encendidas durante su examen.

A continuación, se anexa la parte de evaluación del Programa Analítico basado en competencias de Matemáticas superior.

\begin{tabular}{|c|l|c|c|}
\hline PIA & $\begin{array}{l}\text { Reporte de resolución de problemas de aplicación } \\
\text { matemática en el área fisicoquímica o biológica. }\end{array}$ & $20 \%$ & $20 \%$ \\
\hline & Total & $\mathbf{1 0 0 \%}$ & $\mathbf{1 0 0 \%}$ \\
\hline
\end{tabular}

\section{IV- RESULTADOS}

Comparando las calificaciones del semestre virtual agosto - diciembre 2020 vs dos semestres anteriores agosto - diciembre 2019 y agosto - diciembre 2018 se observó que el número de alumnos reprobados en primera oportunidad fue solamente de $5 \%$ (4 de 80 ) para el semestre virtual y para el curso del 2019 fue el $31 \%$ (25 de 80 ) y del 2018 un 35\% (28 de 80).

A los alumnos del semestre virtual se les aplicó una encuesta y éstos fueron los resultados:

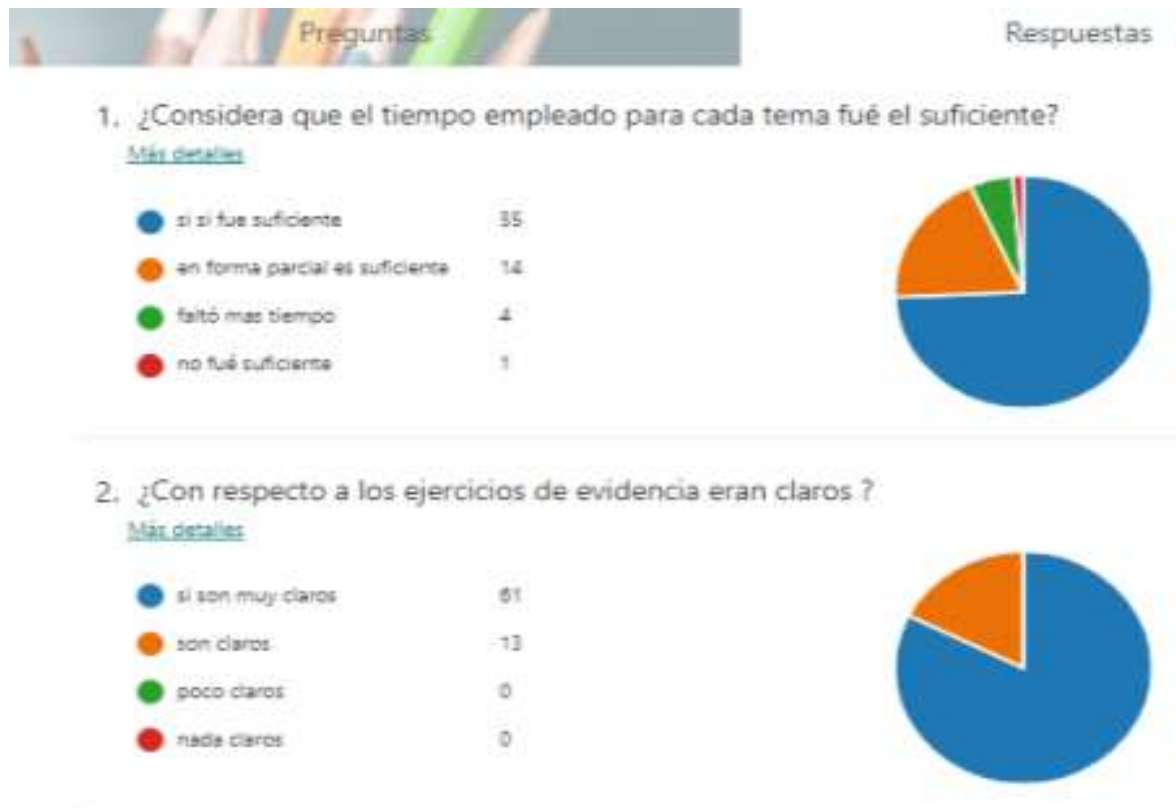


3. ¿En los quiz los reactivos están relacionados con los temas revisados? Matsertitis
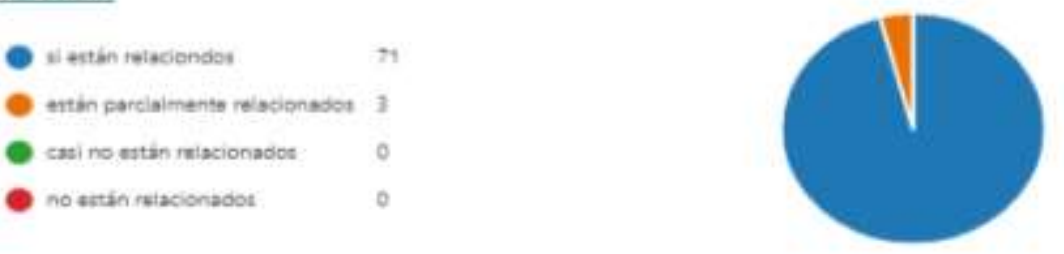

4. ¿En los exámenes parciales considera que si corresponden las preguntas a los temas revisados? Meterales

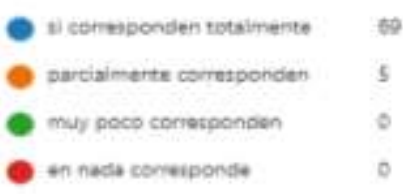

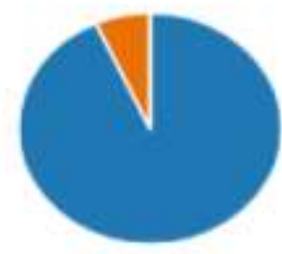

5. En los temas relacionados con ecuación de Equilibrio químico Nerts, Hasselbach. Calisus y extracción de liquidos ¿Cómo fué su comprensión y/o calificación? Manderales
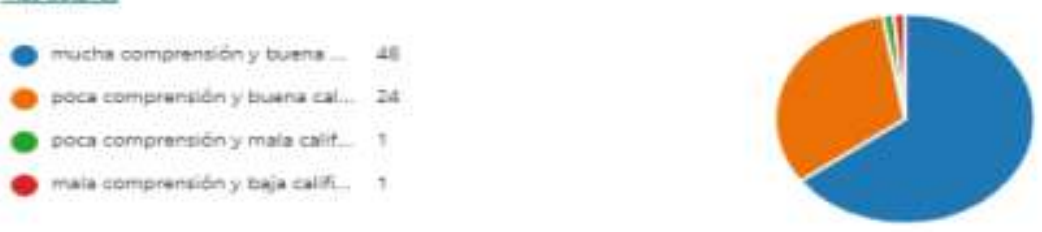

6. ¿Cómo considera la utifidad de realizar los ejercicios por equipos en sus respectivas salas en Teams?

$$
\text { Mistemeses: }
$$

$$
\begin{aligned}
& \text { C. muy util } \\
& \text { C viel } \\
& \text { 2) poco ints } \\
& \text { C nadu unil }
\end{aligned}
$$


9. Cómo evalúa el uso de nearpod en el caso de los simuladores o la de climb to top para las actividades de ejercicios

Matidealen:

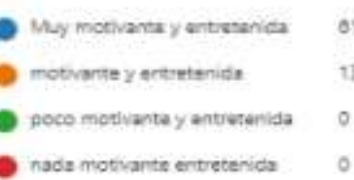

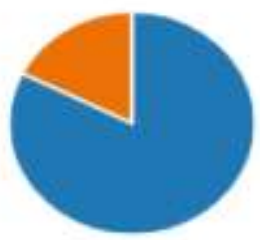

10. Comentarios respecto a la UA de matemáticas Manderalm

\begin{tabular}{|c|c|}
\hline Id. & Respuestas \\
\hline \multicolumn{2}{|r|}{ Gracias por la dedicación y el tiempo } \\
\hline 14 & Fue la clase que mas di sfrute durante el semestre, gracias por todo c: \\
\hline 20 & $\begin{array}{l}\text { Me ayudó mucho realizar mapas previos a las clases y las actividades } \\
\text { en nearpod }\end{array}$ \\
\hline 36 & Una de las materias que más disfruté \\
\hline 45 & $\begin{array}{l}\text { A prendi cosas nuevas y reforcé conocimientos que ya tenia de los } \\
\text { temas, también le agradezco mucho a la maestra Judith y a la maestra } \\
\text { Samantha por sus enseñanzas < } 3\end{array}$ \\
\hline 42 & $\begin{array}{l}\text { Disfruté mucho de las clases y de cómo fue transmitido el } \\
\text { conocimi ento }\end{array}$ \\
\hline 7 & $\begin{array}{l}\text { La UA de matemáticas fue una materia muy buena y considero que } \\
\text { todo esto fue posible gracias a la Dra. Judith y a la Maestra Samantha. } \\
\text { Fue una experiencia muy buena debido a que siempre hubo un } \\
\text { compromiso por parte de ellas y por parte de los al umnos. }\end{array}$ \\
\hline 4 & La impartición del curso fue muy buena \\
\hline 46 & Muy buena materia y exelentes maestras, hacen que di sfrutes la cl ase \\
\hline 43 & me gusta la manera en que se trabaja \\
\hline 41 & Todo bien todo perfecto unu \\
\hline 34 & $\begin{array}{l}\text { En algunos que dice poco entendimiento o asi no es como tal poco, } \\
\text { sino mas o menos solo que no estaba esa opción. Graciaspor todo } \\
\text { maestra }\end{array}$ \\
\hline 12 & $\begin{array}{l}\text { Excelente unidad de aprendizaje, catedráticos muy bien preparados, } \\
\text { consejos para las demás actividades de aprendi zaje y motivación para } \\
\text { seguir adelante. Gracias por todo su esfuerzo en está nueva modalidad, } \\
\text { por su preparación previa a clase y hacer que aprendamos a pesar de } \\
\text { las circunstancias }\end{array}$ \\
\hline 30 & Mi clase favorita, las explicaciones eran claras asi como los ejercicios \\
\hline 33 & excelentes maestras:) \\
\hline 40 & $\begin{array}{l}\text { Me gusto mucho la dinámica de los mapas y el tiempo de los exámenes } \\
\text { fue el adecuado para terminar bien el mismo. }\end{array}$ \\
\hline 6 & a mi me pareció muy interesante y atractivo \\
\hline 35 & $\begin{array}{l}\text { Creo que el hecho de tener una maestra que nos explica correctamente } \\
\text { y si no entendemos al go se regresa a explicar, ayuda mucho al } \\
\text { desempeño en la unidad de aprendizaje. }\end{array}$ \\
\hline 28 & $\begin{array}{l}\text { La explicación de los temas fue muy buena, me hubiera gustado mas } \\
\text { ejercicios para practicar más }\end{array}$ \\
\hline 2 & Una exelente doctora temas muy bien explicados y nos al enta a seguir a \\
\hline 8 & Se me hizo muy fácil Gracias a la maestra ludith y Samantha \\
\hline 25 & Una UA muy útil y con maestras mury atentas y dedicadas) \\
\hline 16 & Me gustó mucho su forma de enseñar \\
\hline 37 & Muy interesante la materia. \\
\hline 21 & Muy buenas profesoras \\
\hline 23 & $\begin{array}{l}\text { Muchas gracias por su tiempo, es de los mejores profesores que he } \\
\text { conocido en estos años que llevo como estudiante. }\end{array}$ \\
\hline
\end{tabular}


Figura 1. A continuación, se muestran algunos ejemplos de las actividades en la plataforma Nearpood

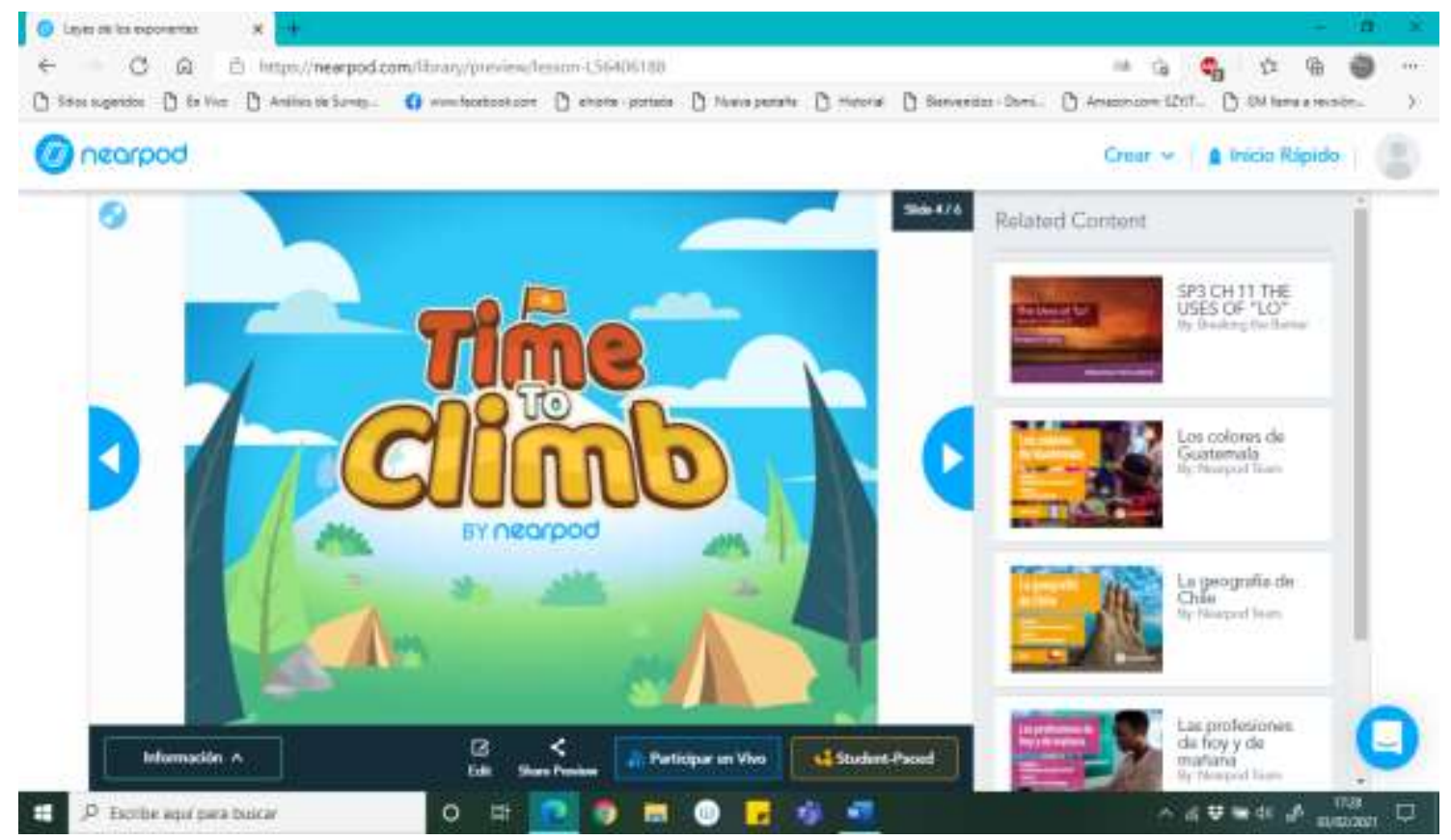

Figura 2. Formato de preguntas del Time to Climb

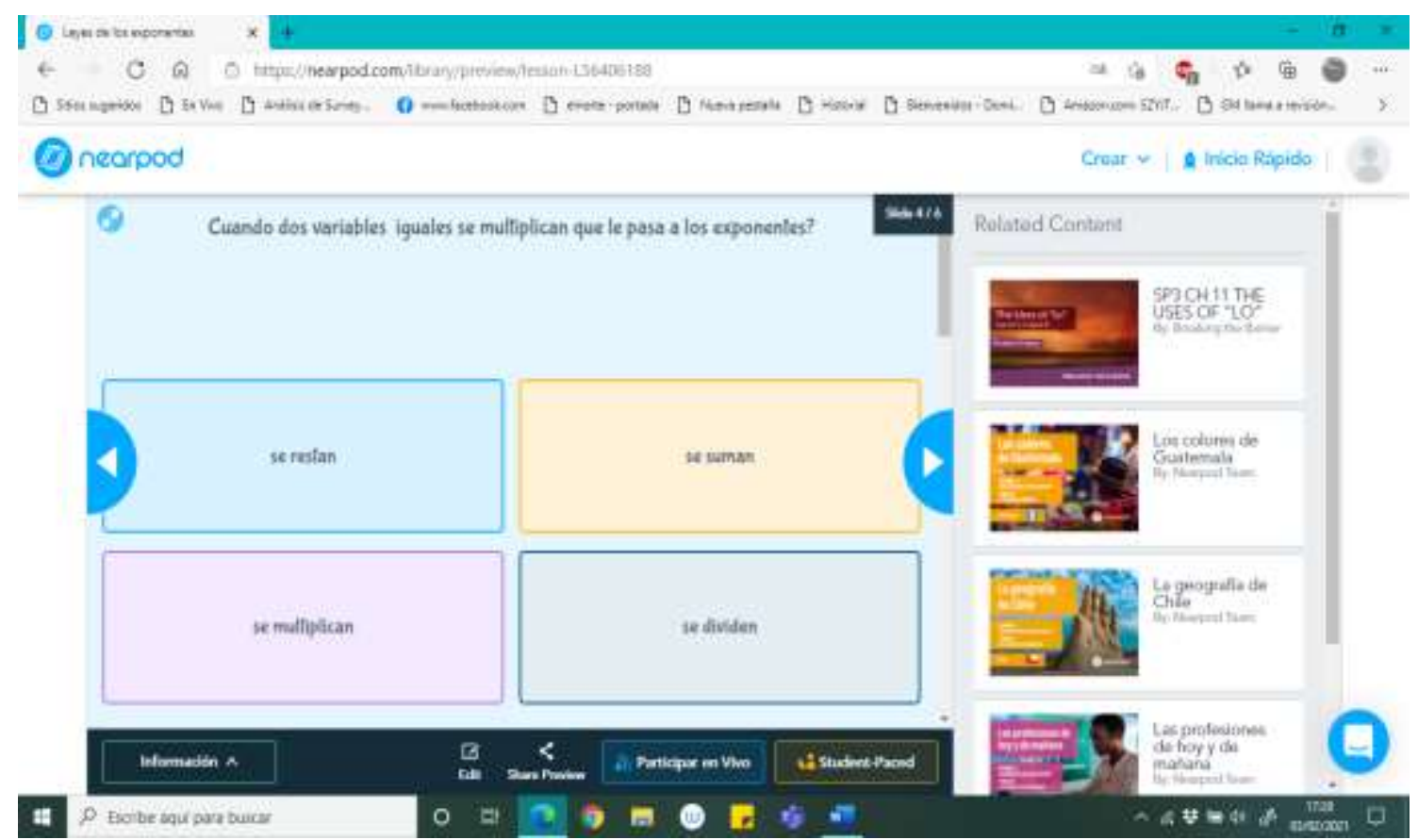

Ciencia Latina Revista Científica Multidisciplinar, Ciudad de México, México. ISN 2707-2207 / ISSN 2707-2215 (en línea), setiembre-octubre, 2021, Volumen 5, Número 5. https://doi.org/10.37811/cl_rcm.v5i5.895 p.8157 
Tabla 2 Se adjuntan las calificaciones obtenidas de los alumnos de la generación 2020 VS LA GENERACIÓN 2018 Y 2019

\begin{tabular}{|c|c|c|}
\hline 2018 & 2019 & 2020 \\
\hline 78.0 & 78.7 & 70.0 \\
\hline 60.9 & 60.7 & 81.0 \\
\hline 70.3 & 89.7 & 87.0 \\
\hline 66.2 & 72.4 & 81.0 \\
\hline 68.5 & 78.8 & 86.0 \\
\hline 66.1 & 82.9 & 76.0 \\
\hline 82.6 & 79.9 & 78.0 \\
\hline 77.2 & 84.6 & 77.0 \\
\hline 75.5 & 69.5 & 71.0 \\
\hline 63.0 & 73.5 & 85.0 \\
\hline 79.8 & 61.9 & 86.0 \\
\hline 70.3 & 76.6 & 83.0 \\
\hline 71.4 & 73.6 & 82.0 \\
\hline 72.8 & 68.9 & 66.0 \\
\hline 73.6 & 87.9 & 67.0 \\
\hline 65.3 & 70.0 & 94.0 \\
\hline 86.0 & 55.9 & 90.0 \\
\hline 66.6 & 45.9 & 84.0 \\
\hline 78.9 & 78.3 & 83.0 \\
\hline 78.9 & 60.1 & 94.0 \\
\hline 79.4 & 79.3 & 81.0 \\
\hline 69.9 & 80.1 & 77.0 \\
\hline 71.4 & 75.1 & 83.0 \\
\hline 79.5 & 62.7 & 80.0 \\
\hline 75.0 & 55.2 & 83.0 \\
\hline 74.5 & 78.5 & 91.0 \\
\hline 86.0 & 71.4 & 85.0 \\
\hline 74.3 & 88.9 & 85.0 \\
\hline 71.7 & 74.3 & 83.0 \\
\hline 74.2 & 73.1 & 84.0 \\
\hline 78.8 & \begin{tabular}{|l|l}
83.3 \\
\end{tabular} & 86.0 \\
\hline 89.0 & 72.5 & 77.0 \\
\hline 79.4 & 87.7 & 78.0 \\
\hline 59.3 & 74.2 & 73 \\
\hline 75.8 & 84.3 & 90.0 \\
\hline 76.0 & 77.6 & 87.0 \\
\hline 76.8 & 24.8 & 81.0 \\
\hline 81.3 & 73.3 & 77.0 \\
\hline 76.6 & 11.0 & 91.0 \\
\hline
\end{tabular}




\begin{tabular}{|c|c|c|}
\hline 76.4 & 51.3 & 92.0 \\
\hline 82.3 & 76.1 & 80.0 \\
\hline 71.7 & 72.6 & 91.0 \\
\hline 83.9 & 62.8 & 86.0 \\
\hline 79.8 & 33.2 & 86.0 \\
\hline 83.6 & 75.7 & 88.0 \\
\hline 93.8 & 83.1 & 80.0 \\
\hline 81.0 & 77.0 & 69.0 \\
\hline 32.9 & 61.9 & 81.0 \\
\hline 73.6 & 72.5 & 91.0 \\
\hline 81.9 & 89.5 & 90.0 \\
\hline 73.1 & 66.9 & 86.0 \\
\hline 50.3 & 74.4 & 78.0 \\
\hline 74.8 & 69.3 & 74.0 \\
\hline 66.5 & 73.2 & 80.0 \\
\hline 66.8 & 86.1 & 63.0 \\
\hline 78.1 & 81.5 & 91.0 \\
\hline 75.1 & 85.7 & 84.0 \\
\hline 78.9 & 52.5 & 74.0 \\
\hline 87.7 & 75.7 & 83.0 \\
\hline 74.1 & 71.4 & 76.0 \\
\hline 73.3 & 79.4 & 86.0 \\
\hline 64.6 & 64.7 & 86.0 \\
\hline 68.0 & 75.9 & 97.0 \\
\hline 65.8 & 70.5 & 90.0 \\
\hline 78.8 & 65.1 & 86.0 \\
\hline 66.2 & 63.9 & 89.0 \\
\hline 70.9 & 87.4 & 82.0 \\
\hline 80.9 & 65.9 & 85.0 \\
\hline 64.5 & 77.3 & 86.0 \\
\hline 73.1 & 74.7 & 96.0 \\
\hline 67.7 & 69.1 & 92.0 \\
\hline 75.4 & 59.9 & 94.0 \\
\hline 74.8 & 83.8 & 12.0 \\
\hline 71.5 & 67.8 & 79.0 \\
\hline 77.1 & 85.7 & 90.0 \\
\hline 72.8 & 85.4 & 87.0 \\
\hline \multirow[t]{4}{*}{68.6} & 91.0 & 88 \\
\hline & 59.2 & 88 \\
\hline & 80.1 & 86 \\
\hline & 78.6 & 80 \\
\hline
\end{tabular}




\section{V- DISCUSIÓN}

Se llevó a cabo la comparación del rendimiento de las generaciones 2018 y 2019 que fue llevada de manera presencial vs la generación 2020 la cual fue virtual o en línea. Al observar los resultados, se demuestra un mejor desempeño de los alumnos en base a sus calificaciones y el porcentaje de aprobados, de esta manera, se recomienda considerar que muy posiblemente se encuentren mas adecuados a la tecnología y esa es la razón de su éxito.

Con base a la encuesta se observa en los rubros donde se revisan temas relacionados con su carrera y la aplicación de ecuaciones de primer grado y despejes que presentan cierto grado de dificultad hacer esa correlación, es un área de oportunidad para remediar También en la encuesta manifiestan que el uso de las salitas virtuales es bueno, pero no excelente, seguramente por que el tiempo en el que el docente visita las salas es muy reducido, se tendrá que considerar dar más tiempo a esta actividad o contar con mas de dos docentes durante la sesión.

\section{VI- CONCLUSIÓN}

Se logró comparar el desempeño académico de alumnos de Matemáticas Superior de la carrera de Químico Clínico Biólogo al llevar a cabo la clase de manera presencial y virtual, mostrando mejores resultados y aprendizaje cuando se llevó a cabo de manera virtual mediante el uso de plataformas digitales. Se propone de manera importante aumentar el uso de plataformas digitales didácticas que acompañen el mejor aprendizaje del alumno.

No hay que olvidar que el profesor es un elemento esencial para mejorar la calidad de los procesos educativos en la metodología a distancia y virtual, en la medida que interactúe con sus estudiantes de manera empática, proactiva, motivante y dialógica a fin de favorecer la asimilación del nuevo conocimiento. Su intervención ha de estimular y orientar al estudiante, por medio de situaciones didácticas de aprendizaje y de diversas estrategias que lo ayudan a superar las dificultades encontradas al abordar una situación problema.(5)(15)

\section{VII- BIBLIOGRAFIA}

Experiencia Docente en un Curso Cero Semipresencial Alejandre M Departamento De

Matemática aplicada Universidad de Zaragoza
www.unizar.es/eees/innovacion06/COMUNIC_PUBLI/BLOQUE_III/CA 
Enseñar Matemáticas en tiempos de Pandemia Marquez G nexos.com Junio 2020 https://educacion.nexos.com.mx/?p=2353

$\begin{array}{llllllll}\text { Tecnicas } & y & \text { Estrategias de Enseñanza Virtual } & \text { Oct } & \text { 17, } & 2017\end{array}$ elearningmasters.galileo.edu/2017/10/17/tecnicas-y-estrategias-de...

Evaluación Del Aprendizaje En Ambientes Virtuales Cano C X CONGRESO NACIONAL DE INVESTIGACIÓN EDUCATIVA | área 7: entornos virtuales de aprendizaje 2005 comie.org.mx/congreso/memoriaelectronica/v10/pdf/area_tematica_...

El Rol del Docente de Matemáticas en Educación a Distancia y Virtual Universitaria Mendoza

https://encuentros.virtualeduca.red/storage/ponencias/peru2019/_. 2019

Laboratorio virtual de matemáticas como estrategia didáctica para fomentar el pensamiento lógico TORRES S Revista Academia y Virtualidad 8(2): 73-84, 2015

La enseñanza de las matemáticas requiere una urgente reestructuración, señala nuevo reporte Observatorio Instituto para El Futuro de la Educación ITESM Paulette Delgado Julio 2020

Aprendizaje virtual de las matemáticas Angela Juan Revista de Universidad y sociedad del conocimiento Universities and Knowlegde Society Journal enero 2012

El papel de los exámenes formativos digitales en el aprendizaje virtual de matemáticas: un estudio de Caso en los Países Bajos Dirk T Templeaar Revista de Universidad y sociedad del conocimiento Universities and Knowlegde Society Journal enero 2012

Conocimientos destrezas y competencias un modelo para aprender matemáticas en un entorno virtual Giovannina Albano Revista de Universidad y sociedad del conocimiento Universities and Knowlegde Society Journal enero 2012

Los cuestionarios del entorno Moodle: su contribución a la evaluación virtual formativa de los alumnos de matemáticas de primer año de las titulaciones de Ingeniería Monica Blanco Universities and Knowlegde Society Journal enero 2012

CUYPERS, Hans (2012). Teaching Mathematics Online: Emergent Technologies and Methodologies, editado por Ángel A. Juan, María A. Huertas, Sven Trenholm y 
Cristina Steegmann» [reseña en línea]. Revista de Universidad y Sociedad del Conocimiento (RUSC). vol. 9, n. ${ }^{\circ}$, págs. 184-189

La enseñanza de las matemáticas: una reflexión sobre su transformación necesaria en tiempos de contingencia Sandra Montserrat Universidad Autónoma de Yucatán Mérida, Yucatán a 30 de junio de 2020

Hacer matemáticas en tiempos de pandemia. Reflexiones para volver a la escuela María Laguna Educación Futura 3 de junio 2020

Impulsando la educación matemática a través de la tecnología durante y después de la pandemia Eleana Ortiz Blog BID mejorando vidas septienbre 24 del 2020 